\title{
Three-Way Doherty Power Amplifier Using Class-F Amplifier for More Efficient Mobile Communications
}

\author{
Ahmed M. Abdulkhaleq ${ }^{1}$, Maan A. Yahya ${ }^{2}$, Maryam Sajedin ${ }^{4}$, Yasir Al-Yasir ${ }^{3}$, Naser \\ Ojaroudi Parchin ${ }^{3}$, Ashwain Rayit ${ }^{1}$, Issa T.E. Elfergani ${ }^{4}$, Raed A. Abd-Alhameed ${ }^{3}$ and \\ Jonothan Rodriguez ${ }^{4}$ \\ \{a.abd@sarastech.co.uk\} \\ ${ }^{1}$ SARAS Technology, Leeds, UK \\ ${ }^{2}$ Ninevah Technical Institute, Northern Technical University, Mosul, Iraq \\ ${ }^{3}$ School of Engineering, Design and Technology, University of Bradford, Bradford, UK. \\ ${ }^{4}$ Mobile Systems Group, Instituto de Telecomunicações, Aveiro, Portugal

\begin{abstract}
An efficient three-way Doherty amplifier is designed and simulated using Cadence Microwave Office software. 6W, 25W and 45W GaN HEMT transistors are used to design three-way Doherty amplifier at $3.4-3.8 \mathrm{GHz}$ band to achieve a $76 \mathrm{~W}$ peak power. Each amplifier was designed to work as a class $\mathrm{F}$ amplifier to improve the efficiency of the designed amplifier. In addition, A new configuration of impedance modulation is used, where a $12.5 \mathrm{~dB}$ gain was obtained over the band, in addition, a $13 \mathrm{~dB}$ back-off efficiency was achieved with 50-60\% drain efficiency. Changing the location of the peaking amplifier or changing the operation sequence of the peaking amplifier can achieve good efficiency at the back-off region. There was an efficiency improvement of $10 \%$ at the back off by utilizing a class $\mathrm{F}$ amplifier comparing with the classical Doherty design.
\end{abstract}

Keywords: Three Way, Doherty amplifier, class F, Efficiency, Back-off, output power.

\section{Introduction}

The wireless mobile communications systems are utilising modulation schemes with high complexity to increase the data rate and to employ the allocated bandwidth effectively due to including additional services and features that attract the user; however, the modulated signal will have an envelope which can be measured by the peak-to-average-power ratio(PAPR) [1]. The efficiency of power amplifiers can be achieved at the saturation region. Nevertheless, for modulated signals with high PAPR, power amplifiers should be operated at the backed-off region that is far from the most efficient point to keep the required linearity performance, however, the efficiency drops sharply [2-10]. The back-off efficiency of power amplifier can be improved using several techniques; among them, the Doherty technique is the easiest, where no digital signal process (DSP) nor additional complex circuity is required.

In this paper, the benefits of using class $\mathrm{F}$ amplifier will be explained in addition to the use of three-way Doherty amplifier for improving and increasing the back-off efficiency, where a new three-way Doherty amplifier configuration will be used. 


\section{Theory of Class F Amplifier}

Much research has been focusing on class $\mathrm{F}$ amplifier. The main difference between the class $\mathrm{F}$ and class $\mathrm{B}$ amplifier is the utilization of the third harmonic on the output voltage, where class $\mathrm{F}$ amplifier efficiency will be improved compared with class B efficiency. By adding the third harmonic component to the voltage output, the voltage shape will look like a square waveform, whereas the current waveform is a truncated sinusoid[11-14]. In this case, the overlapping between the output current and voltage will be reduced. As a result, the dissipated power will be reduced, and the efficiency will be increased. According to the Fourier series, the square waveform can be generated by adding the odd harmonics to the fundamental waveform with certain coefficients' weights. However, in the practical implementation of the power amplifier, considering more harmonics will increase the circuit complexity. Therefore, in this work, the class F power amplifier output voltage design will rely on the combination of DC, fundamental and third harmonic components. The DC power consumption is mainly depending on the conduction angle as shown in the following equation:

$$
P_{D C}=V_{D C} \cdot \frac{I_{\max }}{2 \pi} \cdot \frac{2 \sin \left(\frac{\alpha}{2}\right)-\alpha \cos \left(\frac{\alpha}{2}\right)}{1-\cos \left(\frac{\alpha}{2}\right)}
$$

Where $V_{D C}$ is the supplied drain voltage, $I_{\max }$ is the maximum drain current, $\alpha$ is the current conduction angle and it depends mainly on the DC current:

$$
\alpha=2 \cos ^{-1}\left(\frac{1}{1-\frac{I_{\max }}{I_{D C}}}\right)
$$

Whereas the RF power can be determined using this equation:

$$
P_{R F}=V_{D C} \cdot \frac{I_{\max }}{4 \pi} \cdot \frac{\alpha-\sin \alpha}{1-\cos \left(\frac{\alpha}{2}\right)} \cdot \gamma\left(\varepsilon_{3}\right)
$$

Where $\gamma\left(\varepsilon_{3}\right)$ is the drain voltage ratio between the fundamental and the third harmonic voltage and can be expressed in the following equation:

$$
\gamma\left(\varepsilon_{3}\right)=\frac{1}{\cos w_{0} t+\frac{R_{L, 3} f_{0} I_{3} f_{0}}{R_{L f_{0}} f_{0}} \cos 3 w_{0} t}
$$

As a result, the transistor output voltage can be calculated using the following equation:

$$
V(t)=V_{D C}-V_{f_{0}} \cos \left(w_{0} t\right)-V_{3 f_{0}} \cos \left(3 w_{0} t\right)
$$

By adding the third harmonic to the output voltage, the output power will be increased, so that the efficiency will be increased according to the following equation:

$$
\eta=\frac{P_{R F}}{P_{D C}}=0.5 \cdot \frac{\alpha-\sin \alpha}{2 \sin \frac{\alpha}{2}-\alpha \cos \left(\frac{\alpha}{2}\right)} \cdot \gamma\left(\varepsilon_{3}\right)
$$




\section{Doherty Amplifier Overview}

The Doherty amplifier operation is explained in detail in many materials; [4-9]; however, a short summary will be provided. W. Doherty, in 1936, came up with a new concept for combining two amplifiers outputs [15], Doherty used a quarter-wavelength transmission line and two tube amplifiers. The first amplifer is called carrier amplifeir, whereas the second one called the peaking amplifier, where the first one operates all the time on the other hand, the second amplifier operates only during the load modulation region, at the same time, the bias condition of the carrier amplifier was as class $A B$, whereas the bias condition of the other amplifier was as class $\mathrm{C}$ amplifier [1]. For the impedance inverter, a quarter wavelength transmision line was used as an to invert the impedance amount seen by the carrier amplifier as illustrated in Figure 1.

The characteristic of a transistor can be explored by applying different gate voltages and checking the behaviour of the drain current, as illustrated in Figure 2. The power amplifier load impedance can be determined using (7) assuming that the drain parasitic of the transistor is disregarded:

$$
R_{\text {opt }}=2 \frac{V_{d d}-V_{\text {knee }}}{I_{\max }}
$$

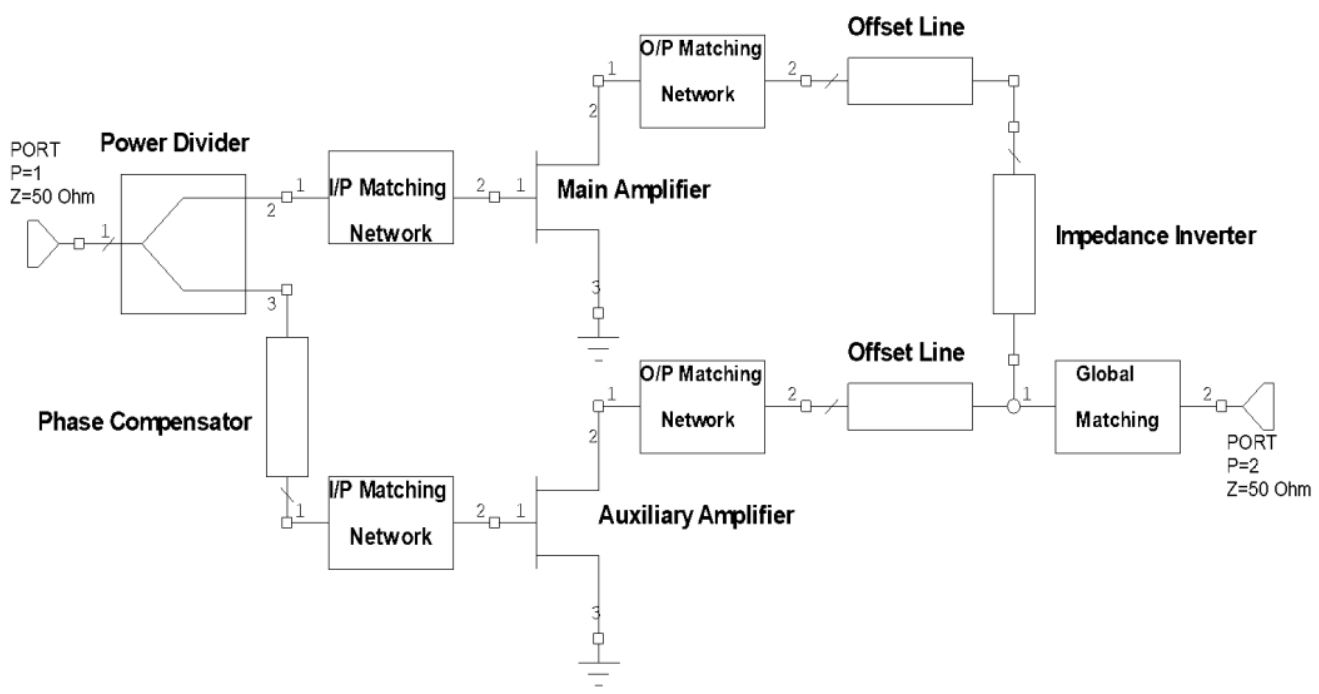

Fig. 1 Doherty amplifier layout [9]. 


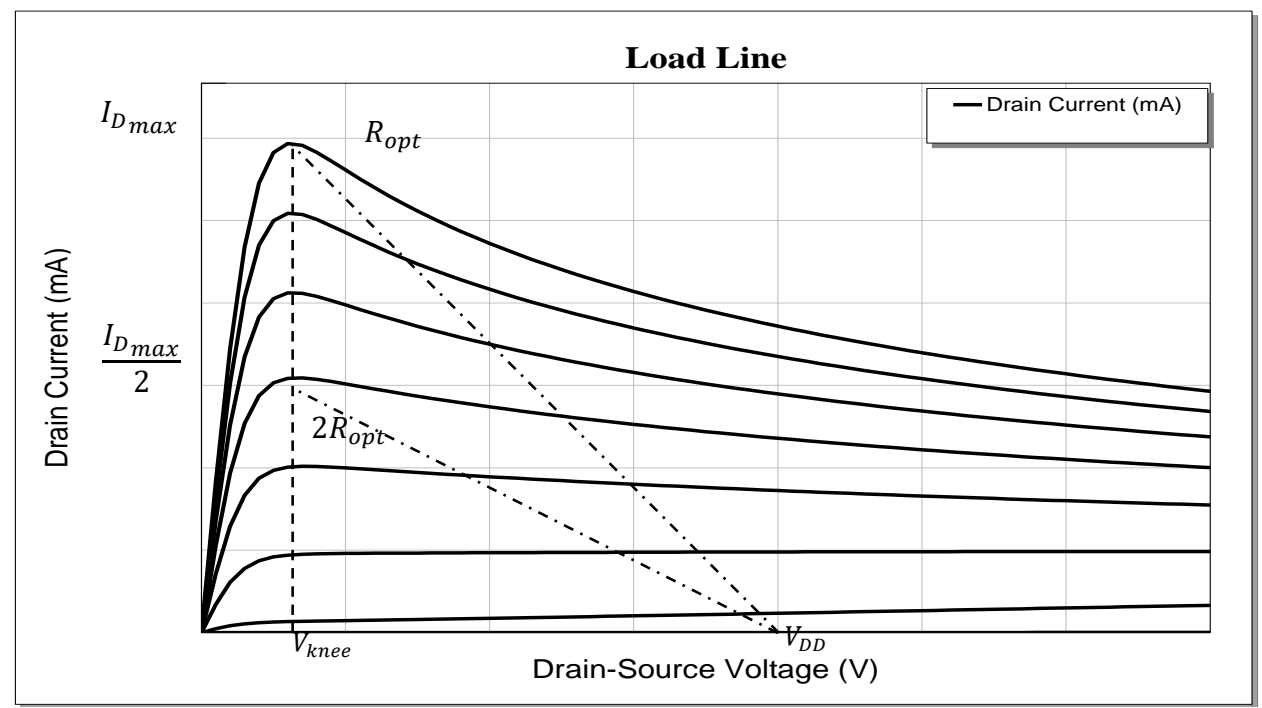

Fig. 2. IV-curves of a transistor [9].

The Doherty amplifier relies on load modulation technique, where its operations can be summarized by two regions of operation assuming both amplifiers are are connected to a load of $25 \Omega$ and matched to $50 \Omega$.

At low input power region, the carrier amplifier is working, nevertheless, due to the quarter wavelength transmission line impedance inversion property, the impedance seen by the carrier amplifier is $100 \Omega$, which is double the optimum load seen by the carrier amplifier, so the first peak efficiency will be seen due to the carrier amplifier saturation.

At the load modulation region, the Doherty operation will be clear, where a current will be injected, to the summing node, by the peaking amplifier, so two current will be contributed to the same load. There will be a reduction from a $100 \Omega$ to $50 \Omega$ of the impedance seen by the carrier amplifier depending peaking amplifier current. However, the saturation of the carrier amplifier continues until its maximum power.

\section{Three-way Doherty Amplifier}

Only 6-dB a peak efficiency at the back-off can be obtained from classical Doherty design assuming both amplifiers have same capabilities. To get more efficiency at further back-off of Doherty power, two different capabilities of power transistors can be used to design asymmetrical Doherty amplifier to accomplish a higher back-off [16, 17]. Another way to increase the back-off efficiency operating region is by utilising more parallel transistors connected via more than one quarter-wavelength line as demonstrated figure 3 . Where one of the transistor acts as the main amplifier and the other two amplifiers act as peaking ones. The main amplifier is responsible for the the first peaking efficiency since it is the only working amplifier and other amplifiers are off. Then, a current will be injected by the first peaking amplifier into the load, where the impedance seen by the main amplifier will be changed. Moreover, additional current will be contributed to the output power from the other peaking 
amplifier in the next region as illustrated in Figure4. Equations (2) to (4) represents the currents amount of each amplifier[4]:

$$
\begin{gathered}
I_{\text {main }}= \begin{cases}I_{m} \frac{V_{\text {in }}}{T_{1} V_{\text {max }}}, & 0<\frac{v_{\text {in }}}{V_{\text {max }}}<T_{1} \\
I_{m}, & T_{1}<\frac{v_{\text {in }}}{V_{\text {max }}}<1\end{cases} \\
I_{\text {Peak } 1}= \begin{cases}0, & 0<\frac{v_{\text {in }}}{V_{\text {max }}}<T_{1} \\
\frac{I_{p 1}}{1-T_{1}}\left(\frac{v_{\text {in }}}{V_{\text {max }}}-T_{1}\right), & T_{1}<\frac{v_{\text {in }}}{V_{\text {max }}}<1\end{cases} \\
I_{\text {Peak } 2}= \begin{cases}0, & 0<\frac{v_{\text {in }}}{V_{\text {max }}}<T_{2} \\
\frac{I_{p 2}}{1-T_{2}}\left(\frac{v_{\text {in }}}{V_{\text {max }}}-T_{2}\right), & T_{2}<\frac{v_{\text {in }}}{V_{\text {max }}}<1\end{cases}
\end{gathered}
$$

Where

$T_{1}<T_{2}$

$I_{m}:$ maximum current of the main amplifier

$I_{p_{1}}$ : maximum current of the first peaking amplifier

$I_{p 2}$ : maximum current of the second peaking amplifier

$T_{1}$ : injecting starting point of the first peaking amplifier

$\mathrm{T}_{2}$ : injecting starting point of the second peaking amplifier

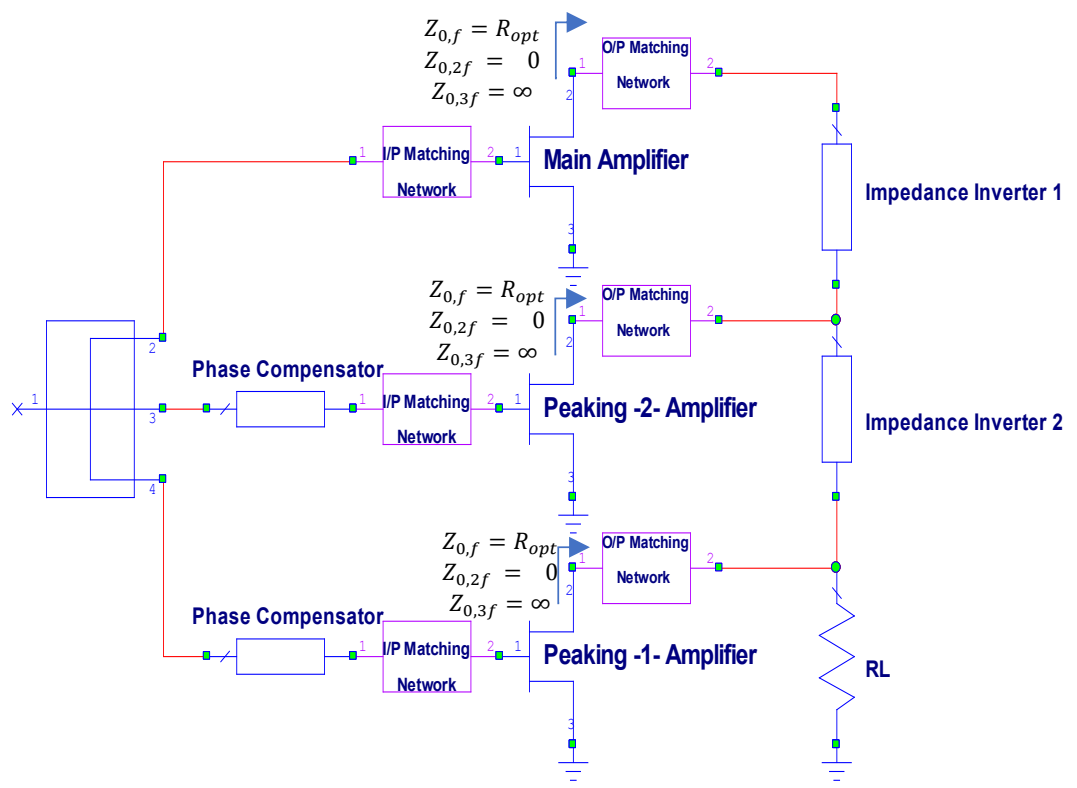

Fig.3. The circuit layout of three-way Doherty amplifier using class-F. 


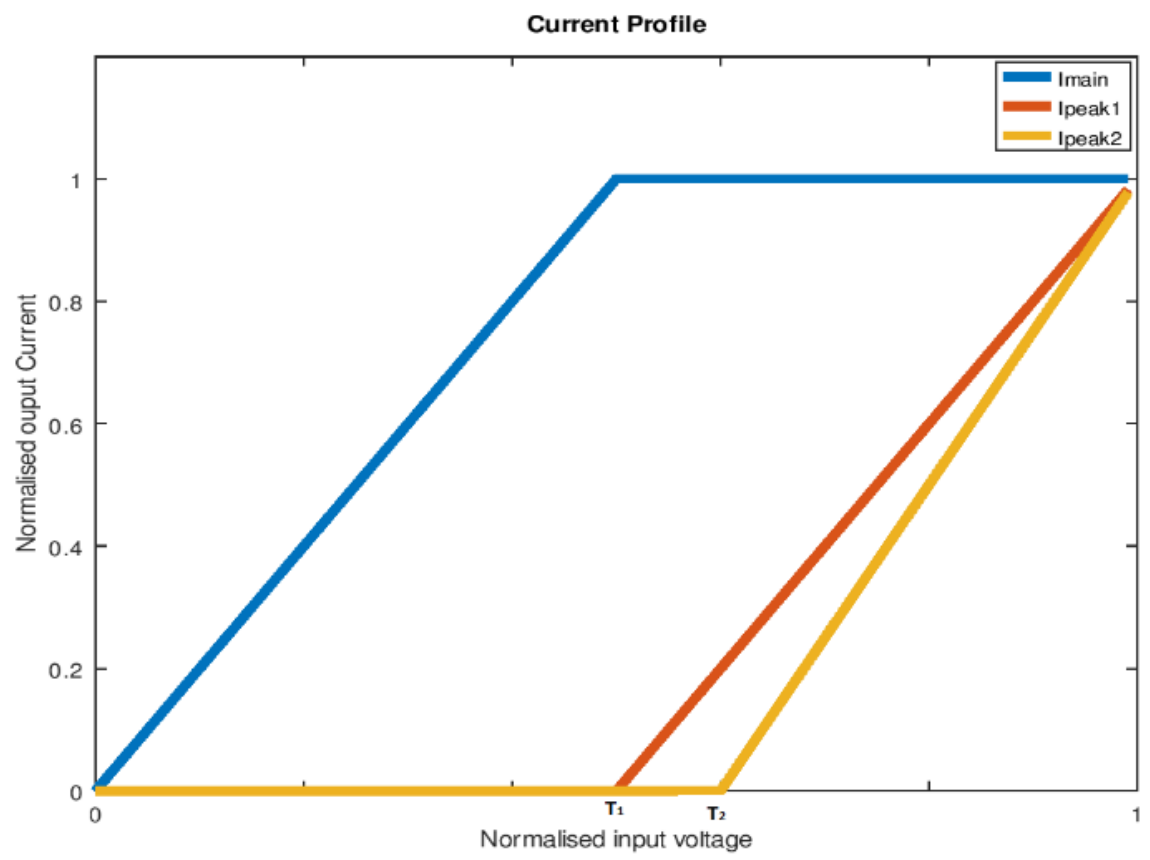

Fig. 4. The 3-way Doherty amplifier current profile.

In this three-way Doherty amplifier circuit, the configration has been modified where the amplifiers orders from the top to the bottom is the main, the second peaking amplifier, then the first peaking amplifier which is located next to the load, two quarter-wavelengths transmission lines are used to connect the three amplifiers to the load. Phase difference compensation was achieved at the input side using additional offset lines to compensate the phase differnce due to the biasing difference and the impedance inversion network.

The proposed three-way Doherty amplifier performance was simulated for two different cases. The first case, when the carrier amplifier is biased as class $\mathrm{AB}$ and the peaking amplifiers as class $\mathrm{C}$, where the second and third harmonics are shorted. Figure 5 shows the performance of the designed amplifier, where 40-50\% efficiency was achieved at $13 \mathrm{~dB}$ back-off with 12.5 $\mathrm{dB}$ gain. In the second case, the designed Doherty amplifier performance utilizing class $\mathrm{F}$ amplifier was simulated, where drain efficiency of 50-60\% was obtained at $13 \mathrm{~dB}$ output backoff, with $12.5 \mathrm{~dB}$ linear gain as shown in Figure 6. Moreover, in both cases, a drain efficiency of $65 \%$ was obtained at the peak power of $48.8 \mathrm{dBm}$.

Moreover, the voltage and current waveform of the designed class $F$ amplifier was simulated as shown in Figure 7. It can be seen that the voltage looks like a square waveform, whereas the current waveform is a truncated sinusoid. There is a small dip in the voltage waveform due to the knee voltage effect. 


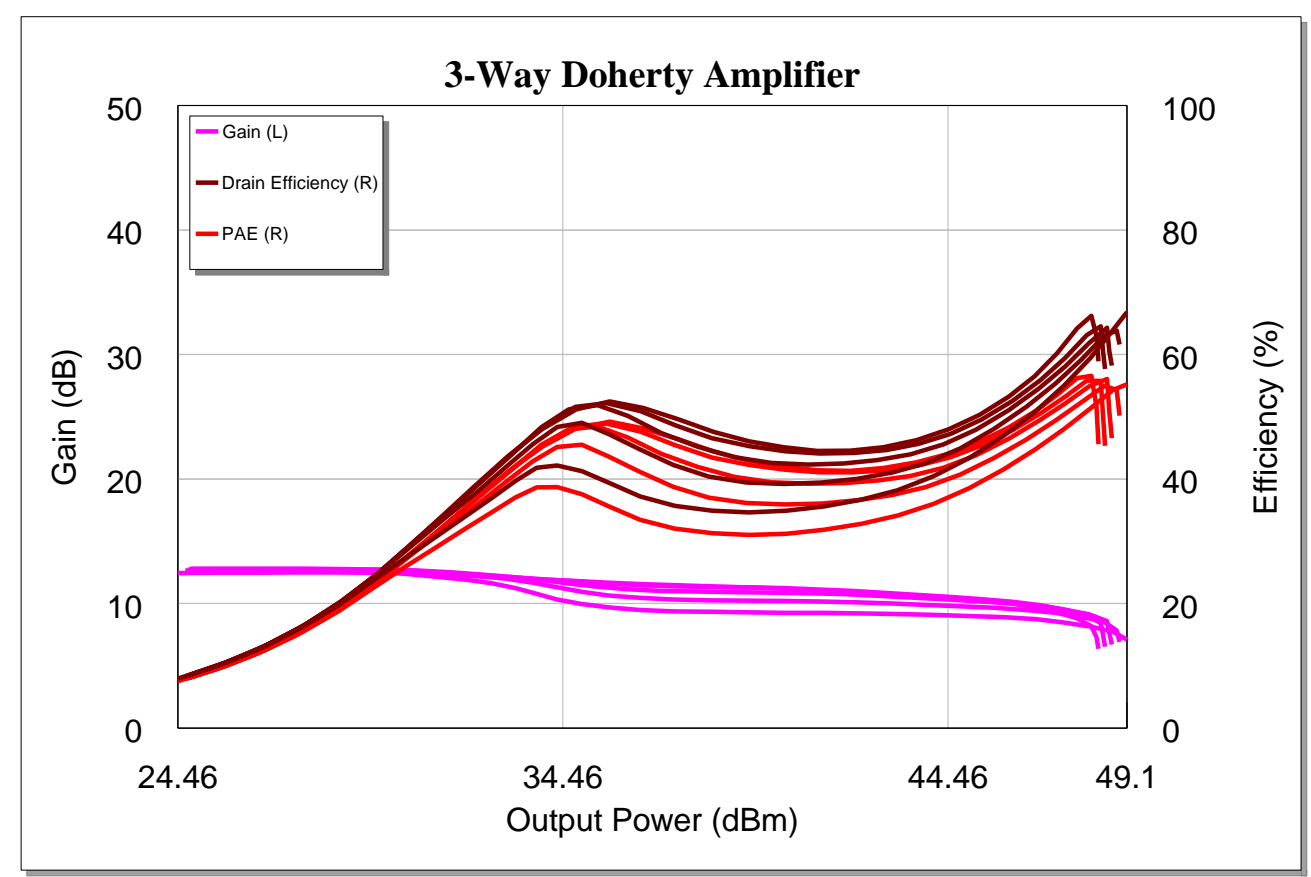

Fig. 5. The performance of three-way Doherty power amplifier Using Class B amplifier for the main and Class C for the peaking amplifiers.

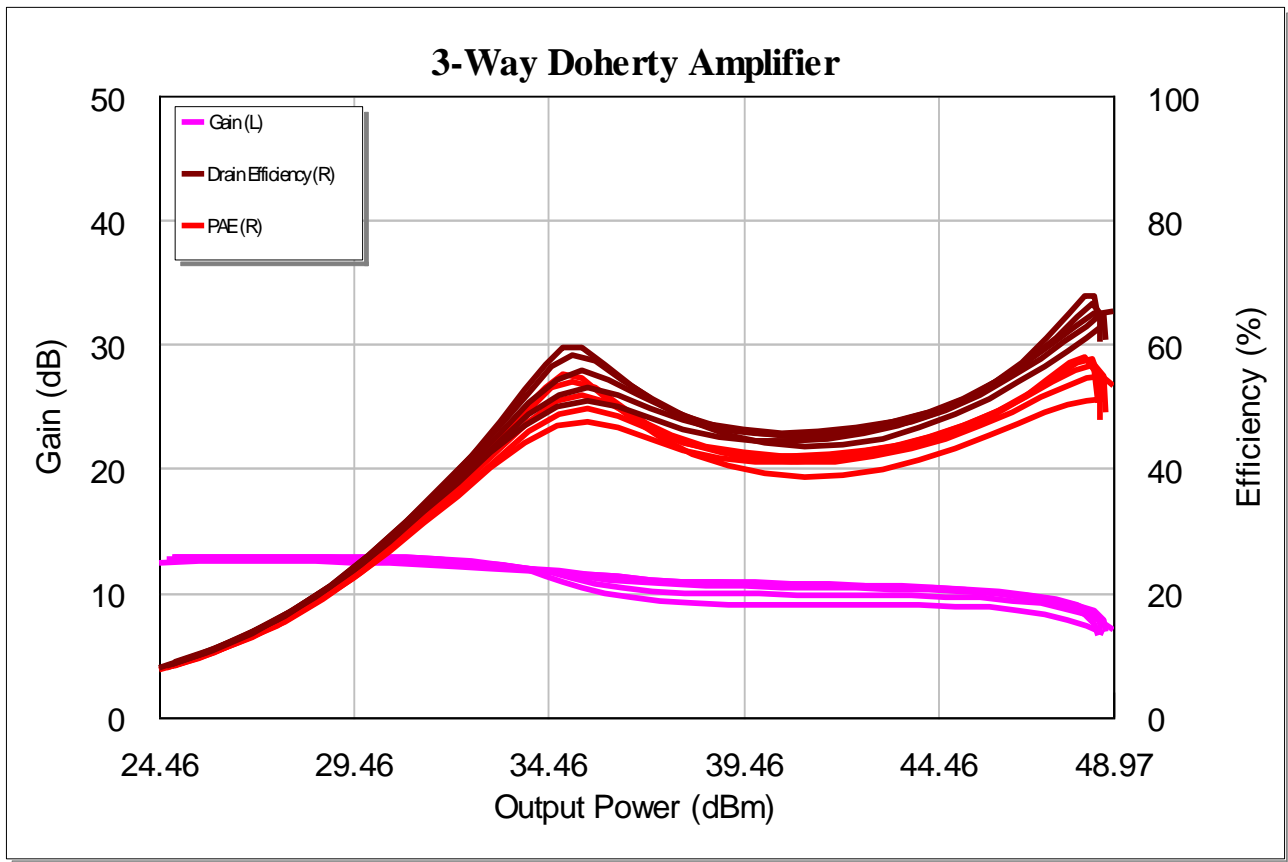

Fig. 6. Designed three-way Doherty power amplifier performance Using Class F amplifier. 


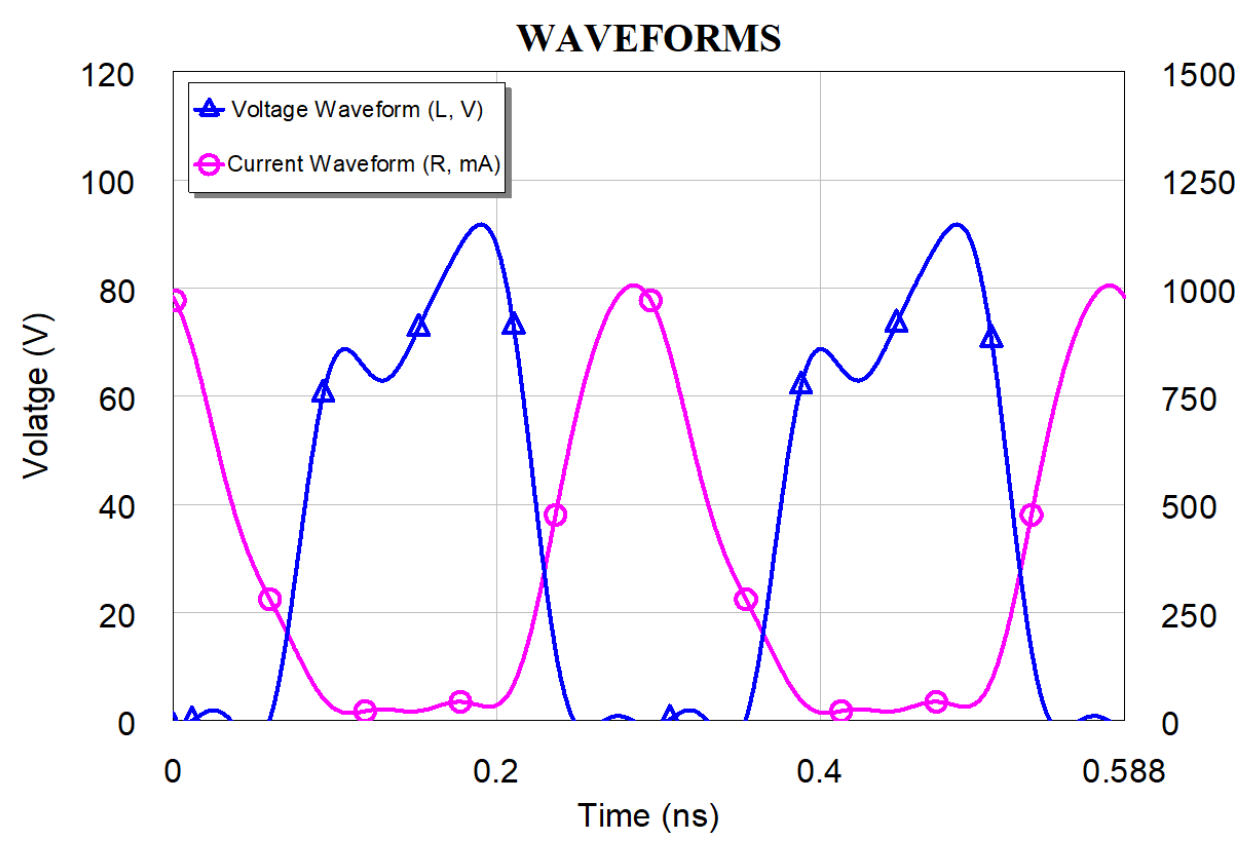

Fig. 7. Simulated class F current and voltage waveforms.

\section{Conclusions}

Three-way Doherty amplifier can be used as a solution for achieving peak efficiency at a deeper back-off power. A configuration of new load modulation has been used for this designed amplifer that can achieve a peak efficiency at a back-off region from the peak power. Changing the peaking amplifiers operation sequence or changing its location can achieve good efficiency at the back-off region; however, the power phase of each amplifier at the summing point should be considered. By using class F amplifier in the three-way Doherty design, the efficiency was improved by about $10 \%$ at the back-off compared to the classical design. Moreover, the gain of the designed amplifier showed a $12.5 \mathrm{~dB}$ over the band, a peak power of $48.8 \mathrm{dBm}$, and a backoff drain efficiency of 55\% was achieved at $13 \mathrm{~dB}$ from the peak power. Knee voltage has an effect of the voltage waveform, where there is a clear dip in voltage waveform.

\section{Acknowledgements}

This paper is funded by the European Union's Horizon 2020 Research and Innovation program under grant agreement H2020-MSCA-ITN-2016-SECRET-722424. 


\section{References}

[1] S. C. Cripps, RF Power Amplifiers for Wireless Communications (Artech House microwave library). Artech House, 2006.

[2] A. M. Abdulkhaleq et al., "18-W Three-Way Doherty Amplifier For 5G Applications," presented at the Antennas and Propagation Conference 2019, Birmingham, UK, 2019.

[3] A. M. Abdulkhaleq et al., "A 70-W Asymmetrical Doherty Power Amplifier for 5G Base Stations," in Broadband Communications, Networks, and Systems, Cham, V. Sucasas, G. Mantas, and S. Althunibat, Eds., 2019// 2019: Springer International Publishing, pp. 446-454.

[4] A. M. Abdulkhaleq et al., "Antennas Impedance Changing on Effect Three-Way Doherty Amplifier for Green RF Communications," presented at the EuCAP 2020, 1520-March-2020, 2020.

[5] A. M. Abdulkhaleq et al., "Doherty Power Amplifier for LTE-Advanced Systems," Technologies, vol. 7, no. 3, 2019, doi: 10.3390/technologies7030060.

[6] A. M. Abdulkhaleq et al., "Load-Modulation Technique for Next Generation Mobile," presented at the 2nd SECRET Workshop - Secure Network Coding for Reduced Energy next Generation Mobile Small Cells,IEEE 5G World Forum, Dresden, Germany, 2019.

[7] A. M. Abdulkhaleq et al., "Noise cancellation for compact MIMO systems," in the 5th International Conference on Internet Technologies and Applications, 2013. [Online]. Available: http://hdl.handle.net/10454/9585

[8] A. M. Yahya et al., "Noise Cancellation for HIPERLAN/2 with Open Loop Transmit Diversity Technique," Inventions, vol. 4, no. 3, 2019, doi: 10.3390/inventions4030046.

[9] A. M. Abdulkhaleq et al., "Recent Developments of Dual-Band Doherty Power Amplifiers for Upcoming Mobile Communications Systems," Electronics, vol. 8, no. 6, 2019, doi: 10.3390/electronics8060638.

[10] A. M. Abdulkhaleq et al., " Load-Modulation Technique Without Using QuarterWavelength Transmission Line," IET Microwaves, Antennas \& Propagation (Accepted).

[11] W. Young Yun, Y. Youngoo, and K. Bumman, "Analysis and experiments for highefficiency class-F and inverse class-F power amplifiers," IEEE Transactions on Microwave Theory and Techniques, vol. 54, no. 5, pp. 1969-1974, 2006, doi: 10.1109/TMTT.2006.872805.

[12] J. Moon, J. Kim, I. Kim, and B. Kim, "Highly Efficient Three-Way Saturated Doherty Amplifier With Digital Feedback Predistortion," IEEE Microwave and Wireless Components Letters, vol. 18, no. 8, pp. 539-541, 2008, doi: 10.1109/LMWC.2008.2001016.

[13] L. Ji, Z. Jianyi, Y. Zhiqiang, and Y. Binqi, "A broadband high efficiency Class-F power amplifier design using GaAs HEMT," in 2015 IEEE International Wireless Symposium (IWS 2015), 30 March-1 April 2015 2015, pp. 1-4, doi: 10.1109/IEEEIWS.2015.7164618.

[14] T. Sharma, R. Darraji, F. Ghannouchi, and N. Dawar, "Generalized Continuous ClassF Harmonic Tuned Power Amplifiers," IEEE Microwave and Wireless Components Letters, vol. 26, no. 3, pp. 213-215, 2016, doi: 10.1109/LMWC.2016.2524989.

[15] W. H. Doherty, "A new high-efficiency power amplifier for modulated waves," The Bell System Technical Journal, vol. 15, no. 3, pp. 469-475, 1936, doi: 10.1002/j.15387305.1936.tb03563.x. 
[16] M. Liu, X. Fang, H. Huang, and S. Boumaiza, "Dual-band 3-way Doherty Power Amplifier with Extended Back-off Power and Bandwidth," IEEE Transactions on Circuits and Systems II: Express Briefs, pp. 1-1, 2019, doi: 10.1109/TCSII.2019.2907481.

[17] M. J. Roberts, "Understanding the 3 level Doherty," in 2016 46th European Microwave Conference (EuMC), 4-6 Oct. 2016 2016, pp. 1357-1361, doi: 10.1109/EuMC.2016.7824604. 